\title{
Ontogenetic and functional modularity in the rodent mandible
}

Rachel A. Menegaza, ${ }^{a,}$, Matthew J. Ravosa ${ }^{b, c, d}$

a Department of Biomedical and Applied Sciences, Indiana University School of Dentistry, 1121 W. Michigan Street, Indianapolis, IN 46202, USA

b Department of Biological Sciences, 100 Galvin Life Science Center, University of Notre Dame, Notre Dame, IN 46556, USA

c Department of Aerospace and Mechanical Engineering, 221 Galvin Life Science Center, University of Notre Dame, Notre Dame, IN 46556, USA

${ }^{a}$ Department of Anthropology, University of Notre Dame, Notre Dame, IN 46556, USA

${ }^{*}$ Corresponding author. Present address: Center for Anatomical Sciences, University of North Texas Health Science Center, Fort Worth, Texas, 76107, USA. E-mail addresses: rmenegaz@iu.edu; rachel.menegaz@unthsc.edu

\section{HIGHLIGHTS}

- Bony responses to diet within the mandible vary by region and by ontogenetic stage.

- Morphogenesis is driven by tooth growth in the corpus, diet in the ramus.

- The morphology of the coronoid-temporalis module reflects early weanling diet.

- The angular-pterygomasseteric module has a longer plasticity window.

- Morphology of the ramus in adults most strongly reflects diet and feeding history.

This is the author's manuscript of the article published in final edited form as:

Menegaz, R. A., \& Ravosa, M. J. (2017). Ontogenetic and functional modularity in the rodent mandible. Zoology. https://doi.org/10.1016/j.zool.2017.05.009 


\section{Abstract}

The material properties of diets consumed by juvenile individuals are known to affect adult morphological outcomes. However, much of the current experimental knowledge regarding dietary effects on masticatory form is derived from studies in which individuals are fed a non-variable diet for the duration of their postweaning growth period. Thus, it remains unclear how intra-individual variation in diet, due to ontogenetic variation in feeding behaviors or seasonal resource fluctuations, affects the resulting adult morphology. Furthermore, the mandible is composed of multiple developmental and functional subunits, and the extent to which growth and plasticity among these modules is correlated may be misestimated by studies that examine non-variable masticatory function in adults only. To address these gaps in our current knowledge, this study raised Sprague Dawley rats $(n=42)$ in four dietary cohorts from weaning to skeletal maturity. Two cohorts were fed a stable ("annual") diet of either solid or powdered pellets. The other two cohorts were fed a variable ("seasonal") diet consisting of solid/powdered pellets for the first half of the study, followed by a shift to the opposite diet. Results of longitudinal morphometric analyses indicate that variation in the mandibular corpus is more prominent at immature ontogenetic stages, likely due to processes of dental eruption and the growth of tooth roots. Furthermore, adult morphology is influenced by both masticatory function and the ontogenetic timing of this function, e.g., the consumption of a mechanically resistant diet. The morphology of the coronoid process was found to separate cohorts on the basis of their early weanling diet, suggesting that the coronoid process/temporalis muscle module may have an early plasticity window related to high growth rates during this life stage. Conversely, the morphology of the angular process was found to be influenced by the consumption of a mechanically resistant diet at any point during the growth period, but with a tendency to reflect the most recent diet. The prolonged plasticity window of the angular process/pterygomasseteric muscle module may be related to delayed ossification and muscular maturation within this module. The research presented here highlights the importance of more naturalistic models of mammalian feeding, and underscores the need for a better understanding of the processes of both morphological and behavioral maturation that follow weaning. 
Keywords: Phenotypic plasticity; Mammals; Fallback foods; Dietary variability

\section{Introduction}

The fundamental goal of functional morphology is to understand the diversity of morphological forms in light of their environmental and behavioral roles. In recent years, phenotypic plasticity has been highlighted in the biological sciences for its potential to shed light on these form-function relationships. Phenotypic plasticity refers to the ontogenetic modulation of a phenotype across an environmental gradient (Stearns, 1989; West-Eberhard, 1993, 2005) and can function as a mechanism for the fine-tuning of form-function relationships across an individual's lifespan (Grant and Grant, 1989; Galis, 1996).

Functional morphologists have long utilized the phenomenon of phenotypic plasticity to explore the link between diet and masticatory form in an experimental setting. By altering diet or even the masticatory apparatus itself, researchers have induced plastic responses through the process of skeletal functional adaptation (sensu Lanyon and Rubin, 1985) and stimulated the development of multiple functional phenotypes within a single laboratory species. These studies, in synthesis with inter-specific comparative work, have demonstrated that the basic principles of functional adaptation of the masticatory system are remarkably similar across mammalian taxa. The material properties of food items are understood to influence jaw adductor activity, jaw kinematics, and feeding behaviors (Crompton, 1986; Weijs et al., 1989; Hylander et al., 1992, 2000, 2005). Increased jaw muscle activity associated with mechanically resistant food items results in elevated peak and cyclical strains in the craniomandibular skeleton (Weijs and de Jongh, 1977; Hylander, 1979, 1988, 1992; Hylander et al., 1992; Herring and Teng, 2000; Ravosa et al., 2007, 2008b, 2015) and, in turn, differential growth and remodeling of hard and soft tissues in the cranium and mandible (Beecher and Corruccini, 1981; Bouvier and Hylander, 1981, 1996; Beecher et al., 1983; Bouvier and Zimny, 1987; Bouvier, 1988; Yamada and Kimmel, 1991; Kiliaridis et al., 1996; 
Nicholson et al., 2006; Ravosa et al., 2007, 2008b, 2010; Menegaz et al., 2009, 2010; Scott et al., 2014a; Franks et al., 2016, 2017; Ravosa et al., 2016).

A common operating condition among these plasticity studies is that the function of interest is held static. For example, studies which experimentally manipulate dietary consistency often rely on a short- or long-term exposure to a stable, homogenous postweaning diet (Beecher et al. 1983; Kiliaridis et al. 1996; Ravosa et al., 2008a; Menegaz et al., 2009, 2010; Ravosa et al., 2016). These homogenous diets do not necessarily reflect the natural variation in diet that occurs due to ontogenetic changes in feeding behaviors (Herring and Wineski, 1986; Hurov et al., 1988; Dardaillon, 1989; Janson and van Schaik, 1993; Bowler and Bodmer, 2011) or to fluctuations in resource availability (Robinson and Wilson, 1998; Marshall and Wrangham, 2007), yet such aspects of dietary variability may exert strong selective pressures on feeding morphology. A select number of studies of joint mechanobiology in rodents have addressed intra-individual variation in dietary composition and found that the masticatory complex of growing individuals may be capable of significant morphological plasticity in response to these dietary changes (Bouvier and Hylander, 1984; Bouvier and Zimny, 1987; Yamada and Kimmel, 1991). Furthermore, adaptive plasticity during early life stages, when growth rates are high, is thought to have an additive influence on underlying growth allometries (Bernays, 1986). In such cases, adult morphology would be strongly affected by the environmental conditions experienced during early life, and modified to a lesser degree by changes in habitat and diet experienced near or after skeletal maturity. Thus, the nature of phenotypic plasticity in the masticatory apparatus has important ramifications for feeding function and performance in mammalian taxa that experience ontogenetic changes in feeding behavior and/or inhabit variable environments.

Moreover, in holding function static in these experiments, we not only underestimate behavioral complexity but skeletal complexity as well. The skeletal morphology of the masticatory apparatus is the product of interactions between genetics, development, and multiple functional pressures (Atchley et al., 1992; Atchley, 1993). Even within a single skeletal element such as the mandible, multiple functional subunits ("modules") exist that all interact uniquely with their associated soft tissues (Moss and Meehan, 1970; Klingenberg et al., 2003a; Zelditch et al., 2008). As feeding behavior changes 
over an individual's life time (through suckling, weaning, and tooth eruption/replacement to the eventual achievement of skeletal maturity), so should the relative importance of functional demands placed on these various morphological modules. The ability of an organism to respond to the environment by means of morphological plasticity may decrease as growth and the rates of bone modeling slow (Hinton and McNamara, 1984; Bertram and Swartz, 1991; Rubin et al., 1992; Pearson and Lieberman, 2004; Hoverman and Relyea, 2007; but see Scott et al., 2014b). However, it is underappreciated to what extent the onset and rate of this decline in plasticity varies among the modules of the masticatory apparatus.

Indeed, the extent to which the growth of various morphological components within the mandible is correlated was addressed previously by Atchley et al. (1992). The authors tested multiple hypotheses of morphogenesis to explain variation in mandibular form between the two main genera of laboratory rodent (Mus and Rattus). Of these hypotheses, two models were considered that potentially explain the differential growth of the mandibular regions in these taxa. The muscle hypertrophy model posits that muscle-bone interactions occurring in the mandibular ramus could drive morphological variation, while the tooth growth model suggests that variation is related to interactions between the teeth and the mandibular corpus. Atchley et al. (1992) found in their study of adult rodents support for all hypotheses except the tooth growth model.

With the muscle hypertrophy and tooth growth models in mind, the present study evaluates the functional sources and the spatial distribution of variation in mandibular morphology at multiple ontogenetic stages within the lifespan of a single species (Rattus norvegicus). The goals of this research were two-fold. The first goal was to investigate the role of intra-individual variation in diet on mandibular morphology. We predicted that feeding behaviors during early, post-weaning life stages would have a disproportionate effect on morphological outcomes in adults due to ontogenetic decline in growth rates. The second goal was to explore longitudinal variation in phenotypic plasticity within the mandible. We predicted that the timing and rates of plasticity responses would vary among mandibular modules, with those modules related to masticatory function (e.g., joint and muscle attachment structures) showing the greatest response during periods in which the individual consumed a more mechanically resistant diet. 
Accordingly, the present study attempted to model the temporal complexity of the material properties of mammalian diets in a laboratory setting. In addition to two treatment groups representing the static homogenous ("annual") diets found in many previous studies, this work also included two variable diet cohorts that experienced a shift in dietary composition during their post-weaning growth period. An "early seasonal" cohort was weaned onto a non-mechanically challenging diet consisting of powdered meal, then switched to a more challenging diet consisting of solid compressed pellets at the mid-juvenile stage. This strategy models the weaning behavior of many mammalian species, which preferentially wean their young during periods of high availability for food items that are easily consumed by young individuals with deciduous or incomplete dentition and a still-growing musculoskeletal masticatory apparatus (Russell, 1984; Di Bitetti and Janson, 2000; Pride, 2005; Eckardt and Fletcher, 2013). As a comparison to the "early seasonal" cohort, a "late seasonal" cohort was weaned onto the challenging diet (compressed pellets), and then switched to the non-challenging diet (powdered meal) at the mid-juvenile stage.

Though the present study encompasses only a single shift in dietary properties, rather than repetitive shifts such as individuals in seasonally variable environments might experience over a longer lifespan, it represents an opportunity to examine how a marked change in dietary composition affects skeletal growth during the important period of growth between weaning and maturity. Additionally, this model accounts only for variation in dietary material properties, and not for the variation in nutritional content that also characterizes many seasonal diets (Conklin-Brittain et al., 1998; Marshall and Wrangham, 2007) and may influence craniomandibular growth (Miller and German, 1999; Fujita et al., 2016). Finally, the present study uses a longitudinal approach to better elucidate the relationship between diet and mandibular morphology across a single individual's lifetime.

\section{Materials and methods}

\subsection{Experimental sample}

All procedures for this project were conducted in accordance with an IACUC-approved protocol. A total of 42 male Sprague Dawley rats (Rattus norvegicus, 
RRID:RGD_5508397) (Berkenhout, 1769) were obtained from Harlan Laboratories (Haslett, MI, USA) as weanlings (22 days old). All animals were housed in AALACaccredited Office of Animal Resources facilities at the Harry S. Truman VA Hospital, University of Missouri for a period of 13 weeks. Weaning was chosen as the starting point for the experimental period because this approximates a shift in masticatory function in the wild and to minimize the confounding influences of postweaning diets other than those included in the present study. The early period of postweaning growth is also when the capacity for phenotypic plasticity is predicted to be greatest (Goldspink, 1970; Hinton and McNamara, 1984; Meyer, 1987; Bouvier, 1988; Bertram and Swartz, 1991; Rubin et al., 1992; Pearson and Lieberman, 2004; Hoverman and Relyea, 2007; Ravosa et al., 2008a). As Sprague Dawley rats reach skeletal maturity between 80 and 91 days (Roach et al., 2003), the sample was raised to the age of 110 days to ensure the completion of skeletal growth. All animals were housed in individual cages to ensure adequate food intake (Bouvier and Hylander, 1984). Body mass for all animals was measured at least twice weekly to monitor intra- and inter-cohort variation in growth and feeding performance. During weeks of dietary shift (weeks 4 and 10), body mass was measured daily. Body mass analyses and behavioral observations confirmed that none of the animals failed to thrive nor did they develop incisor malocclusions at any point during the experimental period. At the end of the experimental period, all animals were euthanized via inhalation of $100 \% \mathrm{CO}_{2}$ from a compressed tank using a $\mathrm{CO}_{2}$ chamber. Bilateral thoracotomy was used as a secondary means of assuring death.

Animals were randomly sorted into four dietary treatment cohorts for the duration of the experimental period (Table 1). All cohorts were fed ad libitum comparable amounts of diets consisting of LabDiet 5001 Rodent Diet (PMI Nutrition International, St. Louis, MO, USA) in either solid pellet or meal/powdered pellet form. The use of the same diet presented in two different consistencies allows for the modification of masticatory behavior and the frequency/intensity of loading while offering comparable nutrition for all animals. Two cohorts were raised on a stable diet of either pellets (cohort 1, "Annual over-use") or meal (cohort 3, "Annual under-use"). The remaining two cohorts were raised on a variable diet consisting of either pellets (cohort 2, "Early seasonal") or meal (cohort 4, "Late seasonal") for the first half of the experimental period and then switched 
to the opposite diet for the remaining weeks. This schedule models a shift in dietary composition as may be experienced due to seasonal variation in the wild, and allows the evaluation of phenotypic plasticity during the optimal growth period.

\subsection{Material properties of experimental foods}

A portable food tester (Darvell et al., 1996; Lucas et al., 2001) was used to assess the material properties of pellets (Wainwright et al., 1976; Vincent, 1992; Lucas, 1994; Currey, 2002). The elastic, or Young's, modulus $(E)$ is the stress/strain ratio at small deformations, characterizing the stiffness or resistance to elastic deformation (pellet $E=$ 13.61 MPa). Toughness $(R)$ is an energetic property describing the work performed propagating a crack through an item (pellet $R=3,325.12 \mathrm{Jm}^{-2}$ ). Hardness $(H)$ is used to quantify indentation (pellet $H=7.25 \mathrm{MPa}$ ).

Due to the specifications of the food tester, it was possible only to measure the material properties of the whole pellets. The meal diet, comprised of ground pellets, primarily differs from whole pellets in the scale of the food particles. Such differences in dietary consistency are known to evoke differences in ingestion behavior, masticatory muscle recruitment, and biomechanical loading in the masticatory apparatus (Bouvier and Hylander, 1981, 1982, 1984; Kiliaridis et al., 1985; Kiliaridis, 1989; Ravosa et al., 2007, 2008 a,b, 2016). Thus, a meal diet represents a shorter preparation/ingestion time with a decrease in masticatory peak and cyclical loads relative to a diet of whole pellets.

\subsection{D imaging and landmark digitization}

Between the ages of 4 and 16 weeks, all animals were imaged weekly using microcomputed tomography $(\mu \mathrm{CT})$ to produce a longitudinal series of three-dimensional (3D) images of the craniofacial skeleton. The Siemens Micro-SPECT/CT unit was operated at $80 \mathrm{kV}$ and $500 \mathrm{~mA}$, with reconstruction using $0.126 \mathrm{~mm}^{3}$ voxels. During imaging, animals were anesthetized via inhalation anesthesia using an isoflurane nonrebreathing anesthetic system at 3.0\% per minute induction rate, and maintained at the $2.5-3.0 \%$ level for the duration of the scan. Body temperature during anesthesia induction, imaging procedure, and recovery period was supported using heating pads and a heat lamp. 
In order to assess morphological differences among cohorts at different ontogenetic stages, 3D landmark data were collected from $\mu \mathrm{CT}$ scans for weeks 4,10 , and 16 . These weeks represent the beginning, middle, and end of the experimental period, respectively. They also represent the three ontogenetic stages encompassed by the experimental period: weaning/prepubescence (weeks 4-7), adolescence (weeks 8-12), and skeletal maturity/adulthood (weeks 13-16). 3D landmarks for the right hemimandible (Fig. 1 and Table 2) were collected for each ontogenetic point using the landmark placement plugin for eTDIPS (Mullick et al., 1999). Landmarks were collected only from $\mu \mathrm{CT}$ scans of acceptable quality; if scans were distorted due to animal movement or technical issues, these scans were excluded. Sample sizes for each cohort by week, less those scans excluded due to quality control issues, are listed in the supplementary Table $\mathrm{S} 1$ in the online Appendix.

A repeatability study $(n=4$, trials $=4)$ was conducted to ensure precision in right-side mandibular landmark placement with resulting standard errors $(0.05-0.57 \mathrm{~mm})$ below $5 \%$ of mean skull length during week 10 (mean $=44.0 \mathrm{~mm}, 5 \%$ of mean $=2.2 \mathrm{~mm}$ ). Visual inspection of landmark accuracy was also performed on individual wireframe models after Procrustes superimposition in Morphologika v2.5 (O'Higgins and Jones, 1998).

\subsection{Morphometric and statistical analyses}

A generalized Procrustes analysis (GPA) was performed on the right hemimandible landmark sets from each ontogenetic point using the Morphologika v2.5 software package (O'Higgins and Jones, 1998). In order to assess variation in hemimandible size, Kruskal-Wallis tests $(\alpha=0.05)$ were used to statistically compare In-transformed centroid sizes among cohorts for each longitudinal point. When a statistically significant difference was detected among cohorts within a given longitudinal point, individual pairwise comparisons were made using the Mann-Whitney U-test. For all statistical tests involving multiple pairwise (inter-cohort) comparisons, relaxed Bonferroni-adjusted $p$-values were used ( $\alpha=0.017$ or $0.05 / 3$ pairwise comparisons per cohort) (Milne and O'Higgins, 2002). 
Two methods were used to evaluate shape variation among the cohorts. In the first, overall differences in the configuration of the hemimandible were assessed using Procrustes distances (Klingenberg et al., 2003a). Pairwise comparisons of Procrustes distances were made using a permutation method (10,000 permutations, adjusted $\alpha=$ 0.017 ) to test the null hypothesis of no difference among the cohort means. In the second method of analyzing morphological variation, canonical variates analyses (CVA) were used to identify the shape differences that best distinguished the dietary cohorts. CVA combines multiple shape variables to produce a small number of canonical variates (CVs) that maximize the differences among cohorts (Albrecht, 1980). The CVs identified by this test are those with the greatest ratios of among-group to within-group variance. CVAs were performed on the hemimandible data sets for weeks 10 and 16 using the MorphoJ software package (Klingenberg, 2011). Procrustestransformed 3D landmarks were used to generate a covariance matrix for each data set. CVAs were then performed on the covariance matrices using dietary cohort as the classification variable. The morphological variables described by the canonical variates were visually assessed using wireframe deformations and lollipop graphs. Visualizations of CVA results in MorphoJ use a reference shape representing a Procrustes distance value of zero (CV score of 0.0 ). Target shapes are derived from the reference shape plus the shape change corresponding to a change in Procrustes distance along the axis of interest (e.g., Procrustes distance of 10.0 is a CV score of +10.0 ). The observations made from these visualizations were then quantitatively confirmed by the statistical comparison of Euclidean distances between the landmarks of interest (Mann-Whitney $U$-test, $\alpha=0.05$; pairwise comparisons using relaxed Bonferroni-adjusted $\alpha=0.017$ ) (Table 3). Morphometric variables for which pairwise comparisons produced nonsignificant $p$-values due to the Bonferroni-adjustment $(0.050<p<0.017)$ are discussed where they are biologically relevant based on the CVA.

\section{Results}

\subsection{Weaning (week 4)}

Although an effort was made to randomly sort individuals among the cohorts, preexisting variation in the size and shape of the right hemimandible was observed during 
week 4. At the time of weaning and before the onset of dietary modification, cohort 1 was observed to have a significantly smaller mandible than cohort 4 (In mandibular centroid size, $p=0.003$ ) (Fig. 2, Tables $S 1$ and S2 in the online Appendix). The Procrustes distances between cohorts 1 and 2, and cohorts 1 and 4 , were also significant, indicating significant differences in overall shape (Fig. 3 and Table S3).

A CVA revealed that two axes best maximized the distance among the cohorts. Axis CV1 accounted for $52.4 \%$ of total variance, and described a significant difference in mandibular and diastema lengths between cohorts 1 and 4 . CV2 accounted for $31.8 \%$ of total variance, and described a significant difference in mandibular condyle length in cohorts 1 and 3 versus cohorts 2 and 4 (Figs. 4 and 5, Table S4). Thus, at the time of weaning, cohort 1 was characterized by a relatively short mandible, short diastema, and a relatively long mandibular condyle.

\subsection{Adolescence (week 10)}

At 10 weeks of age, each of the experimental cohorts had only been exposed to a single diet. Thus, there were two cohorts ( 1 and 2 ) that had been raised on a diet of solid pellets and two cohorts ( 3 and 4 ) that had been raised on a diet of powdered pellets/meal. At this timepoint, there was no significant difference observed among the cohorts in either hemimandibular centroid size (Fig. 2, Tables S1 and S2) or Procrustes distances (Fig. 3 and Table S3).

A CVA revealed that a single CV axis best separated the cohorts by their dietary treatments. CV1 accounted for $88.2 \%$ of total variance, and separated the cohorts raised on pellets ( 1 and 2 ) from those raised on powdered pellets/meal ( 3 and 4$)$. This axis described differences in the mandibular ramus and in toothrow length (Figs. 6 and 7, Table S5). Those cohorts raised on pellets ( 1 and 2) tended to have wider articular processes, greater subcondylar angles formed by the intersection of the articular and angular processes, and shorter rows of cheek teeth.

Two features, mandibular length and diastema length, were identified at the weaning timepoint (week 4) as differentiating cohort 1 from the remaining cohorts. These characters were no longer observed to be significantly different among the cohorts in week 10. However, one character (ramus width) did distinguish cohort 1 from the 
remaining cohorts. (Figs. 6 and 7, Table S5). At the adolescent stage, pellet-fed individuals in cohort 1 tended to have a rostrocaudally wider mandibular ramus than both pellet-fed individuals in cohort 2 and meal-fed individuals in cohorts 3 and 4 .

\subsection{Adulthood (week 16)}

At adulthood, significant differences in hemimandibular centroid sizes were observed between the two seasonal cohorts. Cohort 4, the late seasonal (meal/pellet) treatment, had significantly larger hemimandibles than cohort 2 , the early seasonal (pellet/meal) treatment (Fig. 2, Tables S1 and S2). Furthermore, Procrustes distances revealed significant overall shape differences between the two annual cohorts ( 1 and 3 ), and between the two seasonal cohorts (2 and 4) (Fig. 3 and Table S3).

A CVA revealed that at week 16, two axes could be used to separate the cohorts by dietary treatment. Axis CV1 accounted for $57.3 \%$ of total variance and grouped the cohorts by their early diet, distinguishing cohorts 1 and 2 from cohorts 3 and 4. CV1 described differences in mandibular length and coronoid process morphology (Fig. 8). At adulthood, individuals weaned onto pellets tended to have shorter mandibles and greater mandibular notch angles (formed by the intersection of the articular and coronoid processes) than did individuals weaned onto a diet of meal (Fig. 9 and Table S6).

In contrast, axis CV2 (37.5\% of total variance) grouped the cohorts by their late diet, distinguishing cohorts 1 and 4 from cohorts 2 and 3. CV2 described differences in mandibular ramus and angular process morphology (Fig. 8). Individuals who consumed pellets late in life tended to have dorsoventrally taller (as measured by ramus height, $\mathrm{RH}$ ) and rostrocaudally broader (as measured by coronoid process width, CrW, and ramus width, RW) mandibular rami. Furthermore, individuals who had consumed any pellets during their lifetime tended to have greater subcondylar angles formed by the intersection of the articular and angular processes (Fig. 9 and Table S6).

\section{Discussion}

The mammalian mandible can be broadly divided into two functional regions: the ramus, consisting of the articular process and multiple attachment sites for the major 
masticatory muscles, and the corpus, which supports the teeth (Atchley et al., 1992; Klingenberg et al., 2003a). Within each of these regions, there are multiple distinct modules that have genetic, developmental, and functional underpinnings (Atchley et al., 1985, 1992; Cheverud et al., 1997; Klingenberg et al., 2003a; Fish et al., 2011; Anderson et al., 2014). Significantly, the present study found that the osteogenic response to dietary variation differed between mandibular regions, but also among the modules within these regions. Here we show that the nature of morphological plasticity within these regions and modules is related to function, as well as to the ontogenetic timing of that function and the age of the experimental sample.

\subsection{Regional differences in morphological plasticity}

Results from the present study suggest that the muscle hypertrophy and tooth growth models of mandibular morphogenesis (Atchley et al., 1992) in fact need not be exclusive, but that the relative importance of these models may be determined by the ontogenetic stage of the experimental sample. In the present longitudinal sample, variation in aspects of the mandibular corpus (e.g., toothrow and diastema lengths) was greater among the cohorts in earlier life stages (weaning and adolescence). Laboratory rats are monophyodont, with molar eruption and occlusion, respectively, occurring along the following schedule: first molar, days 19 (pre-weaning) and 23; second molar, days 21 (peri-weaning) and 25; third molar, days 35 (post-weaning) and 40 (Hoffman and Schour, 1940). This schedule was confirmed for the Sprague Dawley rats included in the present study using $\mu \mathrm{CT}$ scans. Periodontal mechanoreceptors mature by day 35 (Nasution et al., 2002), while the molar roots continue to develop through 18 weeks of age (Schour and Massler, 1949). Early life stages (weaning and adolescence) are thus characterized by tooth eruption, root growth, and immature periodontal sensory input. During these stages, the tooth growth model of mandibular morphogenesis explains, at least in part, the variation seen within and among cohorts in the present study.

By comparison, at the adult stage the morphological features that best distinguished the dietary cohorts were exclusively those of the mandibular ramus. These features are related to both overall mandible and ramus size, and more specifically to the structure of attachment sites for masticatory muscles (coronoid and angular processes). In this 
respect, intra-specific variation at the adult stage in the present study more closely resembled the prior results of inter-generic variation among adult rodents (Atchley et al., 1992). In individuals who have attained skeletal maturity, the muscle hypertrophy model explains the majority of morphological variation among the cohorts.

In sum, results from the present study are consistent with previous work which has suggested that the mandibular ramus, particularly the features related to muscle insertion sites and joint structures, is more plastic with respect to variation in feeding behavior than the mandibular corpus, which may be influenced by early growth processes and spatial factors (McFadden et al., 1986; Daegling, 1996; Taylor, 2002; Terhune, 2013). These results are also consistent with the observed trend in human populations for adult mandibular morphology to better predict diet than the morphology of juveniles (Holmes and Ruff, 2011). From an ecomorphological standpoint, this suggests that attempts to reconstruct diet based on mandibular form should preferentially consider the adult morphology of the mandibular ramus, and in particular those characters that are associated with masticatory muscle attachment.

The functional boundaries observed between mandibular regions (e.g., ramus and corpus) are also consistent with identified developmental and genetic boundaries in the mandible (Atchley et al., 1985; Leamy, 1993; Cheverud et al., 1997; Mezey et al., 2000; Klingenberg et al., 2003b; Fish et al., 2011), which separate the "hinge" region (mandibular ramus) from the "cap" region (mandibular corpus) (Fish et al., 2011). Thus, the regional functional variation noted in the present study may be associated with genetic and developmental variation between the temporomandibular joint and masticatory muscle insertion sites in the ramus, and the tooth-bearing structures in the corpus.

\subsection{Modular differences in morphological plasticity}

As with prior studies of the rodent mandible, results from the present research are consistent with the existence of multiple modules within the ramus and corpus regions (Atchley et al., 1992; Klingenberg et al., 2003a; Anderson et al., 2014). In addition to differences in their functional roles, these modules are also distinguished by the ontogenetic timing and duration of their plastic responses to variation in feeding 
behavior. According to the results of the present experiment, which manipulated feeding behavior and masticatory muscle recruitment by means of altering dietary consistency, this phenomenon is most distinct in the mandibular ramus.

At the adolescent stage, morphological features of the articular and angular processes were found to separate the cohorts on the basis of diet. Rats raised (from weaning to adolescence) on a diet of solid pellets had articular processes with a wider base and a greater subcondylar angle than did rats raised on a diet of powdered meal. However, at the adult stage when individuals in the variable diet cohorts had consumed two diets of different material properties, a different series of patterns appeared.

Adult rats could be grouped on the basis of mandibular morphology in three distinct ways: by early diet (from weaning to adolescence), by late diet (from adolescence to adulthood), and by the consumption of mechanically resistant pellets at any point during their lifespan. Mandibular size, as measured by In-adjusted centroid size and mandibular length, tended to group individuals by their early diet, regardless of whether they were in the stable or variable diet cohorts. Similarly, the mandibular notch angle, which describes the orientation of the coronoid process relative to the articular process, was found to separate the cohorts on the basis of early diet. By contrast, overall ramus size (height and width) was found to group cohorts by their late diet. Individuals in both the annual over-use cohort and the late seasonal cohort had taller and wider mandibular rami than did individuals in the annual under-use and early seasonal cohorts. Finally, the subcondylar angle, which describes the orientation of the angular process relative to the articular process, was larger in individuals that had consumed pellets at any point during their lifespan, with a tendency to be greatest in individuals processing pellets as their late diet.

Two functional modules within the region of the mandibular ramus emerge from these results: a coronoid process/temporalis muscle complex and an angular process/pterygomasseteric muscle complex (Hiiemae and Houston, 1971; Atchley and Hall, 1991; Anderson et al., 2014). The tendency of coronoid process morphology, along with overall mandibular size, to reflect the weanling diet suggests that these traits may have an early window for phenotypic plasticity related to the high growth rates during this ontogenetic stage. The ability of these features to significantly change in 
response to feeding behavior may decrease as growth rates slow. In comparison, the window of phenotypic plasticity for the angular process/pterygomasseteric complex appears to be relatively long in duration. Angular process morphology tends to reflect the consumption of a mechanically resistant diet at any stage during the growth period, but with a strong tendency to reflect the latest diet. This may be due in part to the presence of a cartilaginous "cap" on the rodent angular process during early growth, and the processes of chondral modeling which occur at muscular attachment sites in this region (Moss, 1969; Frost, 1994).

The ontogenetic nature of these modules may additionally reflect variation in musculoskeletal function over an individual's lifetime. The transition from suckling to weaning is gradual, with jaw muscle anatomy and jaw movements of juvenile mammals not necessarily identical to those of adults (Herring, 1985). Indeed, laboratory rats are weaned before the complete eruption of their permanent dentition (Hoffman and Schour, 1940; Schour and Massler, 1949), the maturation of their periodontal mechanoreceptors (Nasution et al., 2002) and the maturation of their masticatory muscles (Hurov et al., 1988). Specifically, the superficial masseter and internal pterygoid muscles, both of which attach to the angular process, continue to develop in both size and mechanical advantage after weaning (Hurov et al., 1988). The delayed ability of these muscles to function in an adult-like manner may thus contribute to the extended growth period of their attachment sites. An increased ontogenetic resolution of the relative maturation rates of the masticatory muscles and their impact on masticatory kinematics is needed in order to better understand the nature of juvenile feeding behaviors and morphological plasticity.

\subsection{Pre-existing morphological variation}

It is common procedure in experimental studies of phenotypic plasticity to use samples drawn from a similar genetic background, such as littermates, which are then randomly sorted into treatment groups. The assumption behind this procedure is that it minimizes pre-existing variation in the sample, such that any observed variation can be correlated to the experimental treatment with a greater degree of confidence (Festing and Altman, 
2002). However, the assumption that pre-existing variation has thus been minimized or eliminated is not always validated in experimental studies.

The present study found pre-existing morphological variation at the start of the experiment. Weanling animals in the annual over-use cohort were found to have shorter mandibles, shorter diastemata, and longer condyles than individuals in the remaining cohorts. For most of these characters, the observed pre-existing differences did not persist into the adolescent stage (starting at week 10). These results highlight the fact that standard randomization procedures used in similar experiments may be less effective than assumed. Without a longitudinal approach that characterizes pre-existing variation, there is a risk of incorrectly correlating this pre-existing variation with the experimental treatment(s) if said variation is observed only at later time points. Additionally, increased sample sizes in experimental studies of morphological plasticity will help to reduce the effect of pre-existing variation in cases where it is driven by a few outlying individuals.

\section{Acknowledgments}

We thank Scott Maddux for advice on data analysis, and Beth Brainerd, Jason Organ, David Polly and an anonymous reviewer for helpful comments. Chris Vinyard kindly performed the analyses of food material properties. We greatly appreciate the support of the Biomolecular Imaging Center at the Harry S. Truman VA Hospital. Lastly, we thank Olga Panagiotopoulou and José (Pepe) Iriarte-Diaz for the invitation to contribute

to this special issue on "Determinants of mammalian feeding system design". Funding was provided by the NSF to R.A.M. and M.J.R. (BCS-1061368), and the Wenner-Gren Foundation and the American Society of Mammalogists to R.A.M. Support to R.A.M. was provided by the NSF GRFP, the MU Life Sciences Fellowship Program, and the Bushnell Graduate Education and Research Fund.

\section{Appendix A. Supplementary data}

Supplementary data associated with this article can be found in the online version at doi: \#\#\#. 


\section{References}

Albrecht, G.H., 1980. Multivariate analysis and the study of form, with special reference to canonical variate analysis. Am. Zool. 20, 679-693.

Anderson, P.S., Renaud, S., Rayfield, E.J., 2014. Adaptive plasticity in the mouse mandible. BMC Evol. Biol. 14, 1-9.

Atchley, W.R., 1993. Genetic and developmental aspects of variability in the mammalian mandible. In: J. Hanken, B.K. Hall (Eds.), The Skull, vol. 1. University of Chicago Press, Chicago, p. 207-247.

Atchley, W.R., Hall, B.K., 1991. A model for development and evolution of complex morphological structures. Biol. Rev. 66, 101-157.

Atchley, W.R., Plummer, A.A., Riska, B., 1985. Genetics of mandibular form in the mouse. Genetics 111, 555-577.

Atchley, W.R., Cowley, D.E., Vogl, C., McLellan, T., 1992. Evolutionary divergence, shape change, and genetic correlation structure in the rodent mandible. Syst. Biol. 41, 196-221.

Beecher, R.M., Corruccini, R.S., 1981. Effects of dietary consistency on craniofacial and occlusal development in the rat. Angle Orthod. 52, 61-69.

Beecher, R. M., Corruccini, R.S., Freeman, M., 1983. Craniofacial correlates of dietary consistency in a nonhuman primate. J. Cran. Gen. Dev. Bio., 3, 193-202.

Berkenhout, J., 1769. Outlines of the Natural History of Great Britain and Ireland. P. Elmsly, London.

Bernays, E.A., 1986. Diet-induced head allometry among foliage-chewing insects and its importance for graminivores. Science 231, 495-497.

Bertram, J.E.A., Swartz, S.M., 1991. The 'law of bone transformation': a case of crying Wolff? Biol. Rev. Camb. Philos. Soc. 66, 245-273.

Bouvier, M., 1988. Effects of age on the ability of the rat temporomandibular joint to respond to changing functional demands. J. Dent. Res. 67, 1206-1212.

Bouvier, M., Hylander, W.L., 1981. Effect of bone strain on cortical bone structure in macaques (Macaca mulatta). J. Morphol. 167, 1-12. 
Bouvier, M., Hylander, W.L., 1982. The effect of dietary consistency on morphology of the mandibular condylar cartilage in young macaques Macaca mulatta. Prog. Clin. Biol. Res. 101, 569-579.

Bouvier, M., Hylander, W.L., 1984. The effect of dietary consistency on gross and histologic morphology in the craniofacial region of young rats. Am. J. Anat. 170, 117-126.

Bouvier, M., Hylander, W.L., 1996. Strain gradients, age, and levels of modeling and remodeling in the facial bones of Macaca fascicularis. In: Davidovitch, Z., Norton, L.A. (Eds.), The Biological Mechanisms of Tooth Movement and Craniofacial Adaptation. Harvard Society for the Advancement of Orthodontics, Boston, pp. 407-412.

Bouvier, M., Zimny, M.L., 1987. Effects of mechanical loads on surface morphology of the condylar cartilage of the mandible of rats. Acta Anat. 129, 293-300.

Bowler, M., Bodmer, R.E., 2011. Diet and food choice in Peruvian red uakaris (Cacajao calvus ucayalii): elective or opportunistic seed predation? Int. J. Primatol. 32, 1109-1122.

Cheverud, J.M., Routman, E.J., Irschick, D.J., 1997. Pleiotropic effects of individual gene loci on mandibular morphology. Evolution 51, 2006-2016.

Conklin-Brittain, N.L., Wrangham, R.W., Hunt, K.D., 1998. Dietary response of chimpanzees and cercopithecines to seasonal variation in fruit abundance. II. Macronutrients. Int. J. Primatol. 19, 971-998.

Crompton, A.W., 1986. The evolution of mammalian mastication. In: Wake, D.B., Roth, G. (Eds.), Complex Organismal Functions: Integration and Evolution in Vertebrates. Wiley, New York, pp. 23-40.

Currey, J.D., 2002. Bones: Structure and Mechanics. Princeton University Press, Princeton.

Daegling, D.J., 1996. Growth in the mandibles of African apes. J. Hum. Evol. 30, 315341.

Dardaillon, M., 1989. Age-class influences on feeding choices of free-ranging wild boars (Sus scrofa). Can. J. Zool. 67, 2792-2796. 
Darvell, B.W., Lee, P.K.D., Yuen, T.D.B., Lucas, P.W., 1996. A portable fracture toughness tester for biological materials. Meas. Sci. Technol. 7, 954-962.

Di Bitetti, M.S., Janson, C.H., 2000. When will the stork arrive? Patterns of birth seasonality in neotropical primates. Am. J. Primatol. 50, 109-130.

Eckardt, W., Fletcher, A.W., 2013. Weaning in the Virunga mountain gorilla (Gorilla beringei beringei) - factors causing variation in weaned age. Am. J. Phys. Anthropol. 150, 117 (Abstract).

Festing, M.F.W., Altman, D.G., 2002. Guidelines for the design and statistical analysis of experiments using laboratory animals. ILAR J. 43, 244-258.

Fish, J.L., Villmoare, B., Köbernick, K., Compagnucci, C., Britanova, O., Tarabykin, V., Depew, M.J., 2011. Satb2, modularity, and the evolvability of the vertebrate jaw. Evol. Dev. 13, 549-564.

Franks, E.M., Holton, N.E., Scott, J.E., McAbee, K.R., Rink, J.T., Pax, K., Pasquinelly, A.C., Scollan, J.P., Eastman, M.M., Ravosa, M.J., 2016. Betwixt and between: intracranial perspective on zygomatic arch plasticity and function in mammals. Anat. Rec. 299A, 1646-1660.

Franks, E.M., Scott, J.E., McAbee, K.R., Scollan, J.P., Eastman, M.M., Ravosa, M.J., 2017. Intracranial and hierarchical perspective on dietary plasticity in mammals. Zoology 123, \#\#-\#\#.

Frost, H.M., 1994. Wolff's law and bone's structural adaptations to mechanical usage: an overview for clinicians. Angle Orthod. 64, 175-188.

Fujita, Y., Goto, S., Ichikawa, M., Hamaguchi, A., Maki, K., 2016. Effect of dietary calcium deficiency and altered diet hardness on the jawbone growth: a micro-CT and bone histomorphometric study in rats. Arch. Oral Biol. 72, 200-210.

Galis, F., 1996. The application of functional morphology to evolutionary studies. Trends Ecol. Evol. 11, 124-129.

Goldspink, G., 1970. Morphological adaptation due to growth and activity. In: Briskey, E.J., Cassens, R.G., Marsh, B.B. (Eds.), Physiology and Biochemistry of Muscle as a Food. University of Wisconsin Press, Madison, pp. 521-536.

Grant, B.R., Grant, P.R., 1989. Evolutionary Dynamics of a Natural Population: The Large Cactus Finch of the Galapagos. University of Chicago Press, Chicago. 
Herring, S.W., 1985. The ontogeny of mammalian mastication. Am. Zool. 25, 339-350.

Herring, S. W., Teng, S., 2000. Strain in the braincase and its sutures during function. Am. J. Phys. Anthropol. 112, 575-593.

Herring, S.W., Wineski, L.E., 1986. Development of the masseter muscle and oral behavior in the pig. J. Exp. Zool. 237, 191-207.

Hiiemae, K., Houston, W.J.B., 1971. The structure and function of the jaw muscles in the rat (Rattus norvegicus L.). Zool. J. Linn. Soc., 50, 75-99.

Hinton, R.J., McNamara, J.A., 1984. Effect of age on the adaptive response of the adult temporomandibular joint. A study of induced protrusion in Macaca mulatta. Angle Orthod. 54, 154-162.

Hoffman, M.M., Schour, I., 1940. Quantitative studies in the development of the rat molar. I. The growth pattern of the primary and secondary dentin (from birth to 500 days of age). Anat. Rec. 78, 233-251.

Holmes, M.A., Ruff, C.B., 2011. Dietary effects on development of the human mandibular corpus. Am. J. Phys. Anthropol. 145, 615-284.

Hoverman, J.T., Relyea, R.A., 2007. How flexible is phenotypic plasticity? Developmental windows for trait induction and reversal. Ecology 88, 693-705.

Hurov, J., Henry-Ward, W., Phillips, L., German, R., 1988. Growth allometry of craniomandibular muscles, tendons, and bones in the laboratory rat (Rattus norvegicus): relationships to oromotor maturation and biomechanics of feeding. Am. J. Anat. 182, 381-394.

Hylander, W.L., 1979. The functional significance of primate mandibular form. J. Morphol. 160, 223-240.

Hylander, W.L., 1988. Implications of in vivo experiments for interpreting the functional significance of "robust" australopithecine jaws. In: Grine, F.E. (Ed.), Evolutionary History of the "Robust" Australopithecines. Aldine, Chicago, pp. 55-80.

Hylander, W.L., 1992. Functional anatomy. In: B.G. Sarnat, D.M. Laskin (Eds.), The Temporomandibular Joint. Saunders, Philadelphia, pp. 60-92.

Hylander, W.L., Johnson, K.R., Crompton, A.W., 1992. Muscle force recruitment and biomechanical modeling: an analysis of masseter muscle function during mastication in Macaca fascicularis. Am. J. Phys. Anthropol. 88, 365-848. 
Hylander, W.L., Ravosa, M.J., Ross, C.F., Wall, C.E., Johnson, K.R., 2000. Symphyseal fusion and jaw-adductor muscle force: an EMG study. Am. J. Phys. Anthropol. 112, 469-492.

Hylander, W.L., Wall, C.E., Vinyard, C.J., Ross, C., Ravosa, M.J., Williams, S.H., Johnson, K.R., 2005. Temporalis function in anthropoids and strepsirrhines: an EMG study. Am. J. Phys. Anthropol. 128, 35-56.

Janson, C.H., van Schaik, C.P., 1993. Ecological risk aversion in juvenile primates: slow and steady wins the race. In: M. E. Pereira, L. A. Fairbanks (Eds.), Juvenile Primates: Life History, Development, and Behavior. Oxford University Press, New York, pp. 57-74.

Kiliaridis, S., 1989. Muscle function as a determinant of mandibular growth in normal and hypocalcaemic rat. Eur. J. Orthodont. 11, 298-308.

Kiliaridis, S., Engström, C., Thilander, B., 1985. The relationship between masticatory function and craniofacial morphology: I. A cephalometric longitudinal analysis in the growing rat fed a soft diet. Eur. J. Orthodont. 7, 273-283.

Kiliaridis, S., Bresin, A,. Holm, J., Strid, K.G., 1996. Effects of masticatory muscle function on bone mass in the mandible of the growing rat. Acta Anat. 155, 200205.

Klingenberg, C.P., 2011. MorphoJ: an integrated software package for geometric morphometrics. Mol. Ecol. Resour. 11, 353-357.

Klingenberg, C.P., Barluenga, M., Meyer, A., 2003a. Body shape variation in cichlid fishes of the Amphilophus citrinellus species complex. Biol. J. Linn. Soc. 80, 397408.

Klingenberg, C. P., Mebus, K., Auffray, J.C., 2003b. Developmental integration in a complex morphological structure: how distinct are the modules in the mouse mandible? Evol. Dev. 5, 522-531.

Lanyon, L., Rubin, C.T., 1985. Functional adaptation in skeletal structures. In: M. Hildebrand, D.M. Bramble, K.F. Liem, D.B. Wake (Eds.), Functional Vertebrate Morphology. Harvard University Press, Cambridge, pp. 1-25.

Leamy, L., 1993. Morphological integration of fluctuating asymmetry in the mouse mandible. Genetica 89, 139-153. 
Lucas, P.W., 1994. Categorization of food items relevant to oral processing. In: Chivers, D.J., Langer, P. (Eds.), The Digestive System in Mammals: Food, Form and Function. Cambridge University Press, Cambridge, pp. 197-218.

Lucas, P.W., Beta, T., Darvell, B.W., Dominy, N.J., Essackjee, H.C., Lee, P.K.D., Osorio, D., Ramsden, L., Yamashita, N., Yuen, T.D.B., 2001. Field kit to characterize physical, chemical and spatial aspects of potential primate foods. Folia Primatol. 72, 11-25.

Marshall, A.J., Wrangham, R.W., 2007. Evolutionary consequences of fallback foods. Int. J. Primatol. 28, 1219-1235.

McFadden, L.R., McFadden, K.D., Precious, D.S., 1986. Effect of controlled dietary consistency and cage environment on the rat mandibular growth. Anat. Rec. 215, 390-396.

Menegaz, R.A., Sublett, S.V., Figueroa, S.D., Hoffman, T.J., Ravosa, M.J., 2009. Phenotypic plasticity and function of the hard palate in growing rabbits. Anat. Rec. 292A, 277-284.

Menegaz, R.A., Sublett, S.V., Figueroa, S.D., Hoffman, T.J., Ravosa, M.J., Aldridge, K., 2010. Evidence for the influence of diet on cranial form and robusticity. Anat. Rec. 293A, 630-641.

Meyer, A., 1987. Phenotypic plasticity and heterochrony in Cichlasoma managuense (Pisces, Cichlidae) and their implications for speciation in cichlid fishes. Evolution 41, 1357-1369.

Mezey, J.G., Cheverud, J.M., Wagner, G.P., 2000. Is the genotype-phenotype map modular? A statistical approach using mouse quantitative trait loci data. Genetics 156, 305-311.

Miller, J.P., German, R.Z., 1999. Protein malnutrition affects the growth trajectories of the craniofacial skeleton in rats. J. Nutr. 129, 2061-2069.

Milne, N., O'Higgins, P., 2002. Inter-specific variation in Macropus crania: form, function and phylogeny. J. Zool. 256, 523-535.

Moss, M.L., 1969. Functional cranial analysis of the mandbiular angular cartilage in the rat. Angle Orthod. 39, 209-214. 
Moss, M.L., Meehan, M.A., 1970. Functional cranial analysis of the coronoid process in the rat. Cells Tissues Organs 77, 11-24.

Mullick, R., Warusavithana, S.V., Shalini, V., Pang, P., 1999. Plug-ins: A software model for biomedical imaging and visualization research. http://www.cc.nih.gov/cip/software/etdips.

Nasution, F.H., Toda, K., Soma, K., 2002. Functional maturation of periodontal mechanoreceptors during development in rats. Dev. Brain Res. 139, 307-312.

Nicholson, E.K., Stock, S.R., Hamrick, M.W., Ravosa, M.J., 2006. Biomineralization and adaptive plasticity of the temporomandibular joint in myostatin knockout mice. Arch. Oral Biol. 51, 37-49.

O'Higgins, P., Jones, N., 1998. Facial growth in Cercocebus torquatus: an application of three-dimensional geometric morphometric techniques to the study of morphological variation. J. Anat. 193, 251-272.

Pearson, O.M., Lieberman, D.E., 2004. The aging of Wolff's "law": ontogeny and responses to mechanical loading in cortical bone. Am. J. Phys. Anthropol. 125, 63-99.

Pride, R.E., 2005. Optimal group size and seasonal stress in ring-tailed lemurs (Lemur catta). Behav. Ecol. 16, 550-560.

Ravosa, M.J., Kunwar, R., Stock, S.R., Stack, M.S., 2007. Pushing the limit: masticatory stress and adaptive plasticity in mammalian craniomandibular joints. J. Exp. Biol. 210, 628-641.

Ravosa, M.J., López, E.K., Menegaz, R.A., Stock, S.R., Stack, M.S., Hamrick, M.W., 2008a. Adaptive plasticity in the mammalian masticatory complex: you are what, and how, you eat. In: C.J. Vinyard, M.J. Ravosa, C.E. Wall (Eds.), Primate Craniofacial Biology and Function. Springer Academic Publishers, New York, pp. 293-328.

Ravosa, M.J., López, E.K., Menegaz, R.A., Stock, S.R., Stack, M.S., Hamrick, M.W., 2008b. Using "Mighty Mouse" to understand masticatory plasticity: myostatindeficient mice and musculoskeletal function. Integr. Comp. Biol. 48, 345-359. 
Ravosa, M.J., Ning, J., Costley, D.B., Daniel, A.N., Stock, S.R., Stack, M.S., 2010. Masticatory biomechanics and masseter fiber-type plasticity. J. Musculoskelet. Neuronal Interact., 10, 46-55.

Ravosa, M.J., Scott, J.E., McAbee, K.R., Veit, A.J., Fling, A.L., 2015. Chewed out: an experimental link between food mechanical properties and repetitive loading of the masticatory apparatus in mammals. PeerJ 3, e1345.

Ravosa, M.J., Menegaz, R.A., Scott, J.E., Daegling, D.J., McAbee, K.R., 2016. Limitations of a morphological criterion of adaptive inference in the fossil record. Biol. Rev. Camb. Philos. Soc. 91, 883-898.

Roach, H.I., Mehta, G., Oreffo, R.O.C., Clarke, N.M.P., Cooper, C., 2003. Temporal analysis of rat growth plates: cessation of growth with age despite presence of a physis. J. Histochem. Cytochem. 51, 373-383.

Robinson, B.W., Wilson, D.S., 1998. Optimal foraging, specialization, and a solution to Liem's paradox. Am. Nat. 151, 223-235.

Rubin, C.T., Bain, S.D., McLeod, K.J., 1992. Suppression of the osteogenic response in the aging skeleton. Calcif. Tissue Int. 50, 306-313.

Russell, J.K., 1984. Timing of reproduction by coatis (Nasua narica) in relation to fluctuations in food resources. In: E.G. Leigh, A.S. Rand, D.M. Windsor (Eds.), The Ecology of a Tropical Forest: Seasonal Rhythms and Long-Term Changes. Smithsonian Institution Press, Washington, D.C., pp. 413-431.

Schour, I., Massler, M., 1949. The teeth. In:. E.J. Farras, J.Q. Griffith (Eds.), The Rat in Laboratory Investigation. Hafner Publishing, New York, pp. 104-165.

Scott, J.E., McAbee, K.R., Eastman, M.M., Ravosa, M.J., 2014a. Experimental perspective on fallback foods and dietary adaptations in early hominins. Biol. Lett. 10, 20130789.

Scott, J.E., McAbee, K.R., Eastman, M.M., Ravos, M.J., 2014b. Teaching an old jaw new tricks: diet-induced plasticity in a model organism, from weaning to adulthood. J. Exp. Biol. 217, 4099-4107.

Stearns, S.C., 1989. The evolutionary significance of phenotypic plasticity. Bioscience 39, 436-445. 
Taylor, A.B., 2002. Masticatory form and function in the african apes. Am. J. Phys. Anthropol. 117, 133-156.

Terhune, C.E., 2013. Dietary correlates of temporomandibular joint morphology in the great apes. Am. J. Phys. Anthropol. 150, 260-272.

Vincent, J.F.V., 1992. Biomechanics - Materials. A Practical Approach. IRL Press, Oxford.

Wainwright, S.A., Biggs, W.D., Currey, J.D., Gosline, J.M., 1976. Mechanical Design in Organisms. Princeton University Press, Princeton.

Weijs, W.A., Brugman, P., Grimbergen, C.A., 1989. Jaw movements and muscle activity during mastication in growing rabbits. Anat. Rec. 224, 407-416.

Weijs, W.A., de Jongh, H.J., 1977. Strain in mandibular alveolar bone during mastication in the rabbit. Arch. Oral Biol. 22, 667-675.

West-Eberhard, M.J., 1993. Developmental Plasticity and Evolution. Oxford University Press, New York.

West-Eberhard, M.J., 2005. Developmental plasticity and the origin of species differences. Proc. Natl. Acad. Sci. USA 102, 6543-6549.

Yamada, K., Kimmel, D.B., 1991. The effect of dietary consistency on bone mass and turnover in the growing rat mandible. Arch. Oral Biol. 36, 129-138.

Zelditch, M.L., Wood, A.R., Bonett, R.M., Swiderski, D.L., 2008. Modularity of the rodent mandible: integrating bone, muscles, and teeth. Evol. Dev. 10, 756-768. 
Figure legends
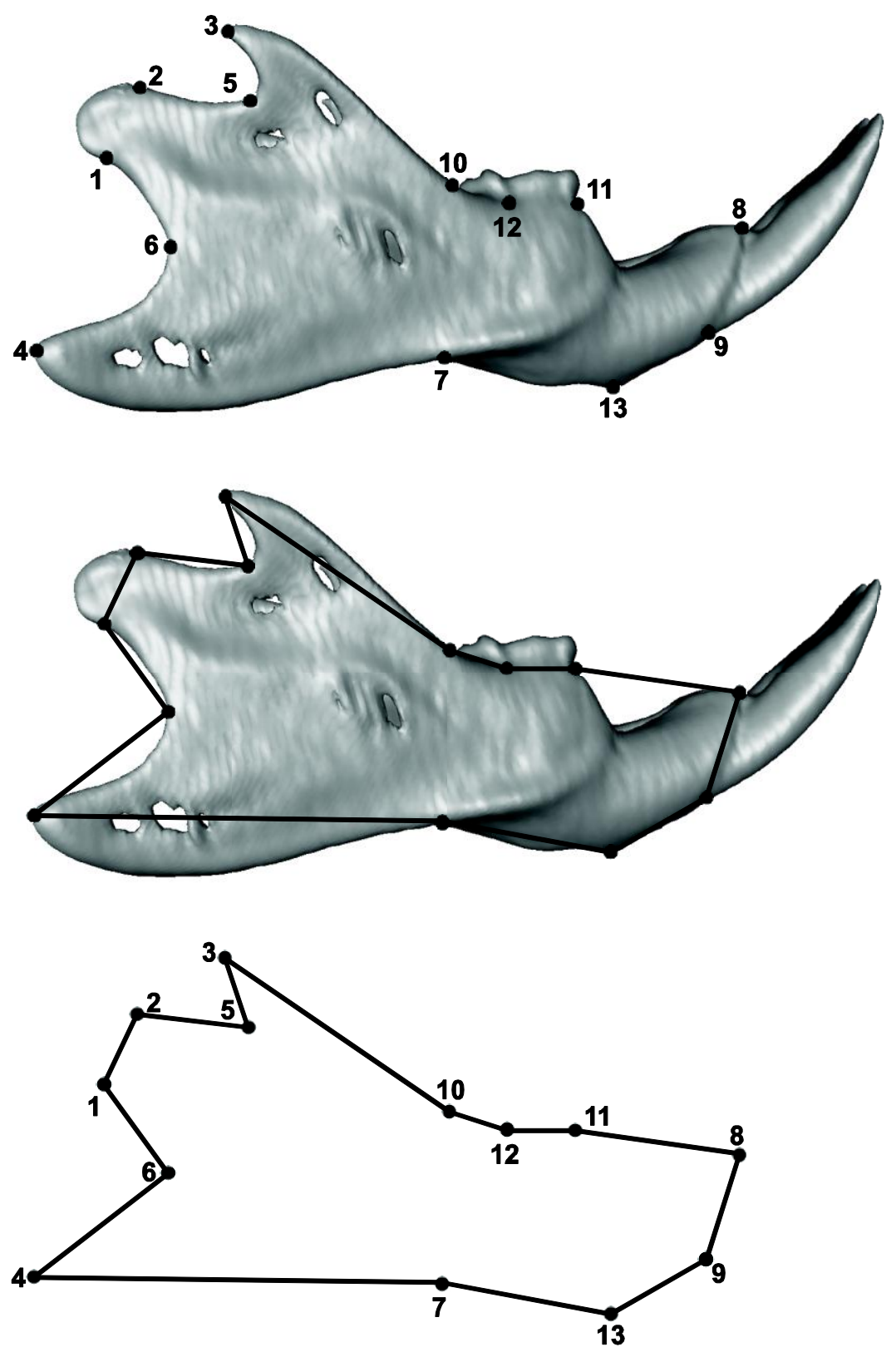

Fig. 1. Landmarks of the right hemimandible. Top, 3D landmarks on a surface model of the rat mandible. Middle, landmarks connected to form a wireframe. Bottom, wireframe of the rat hemimandible used for morphometric analyses. See Table 2 for landmark details. 


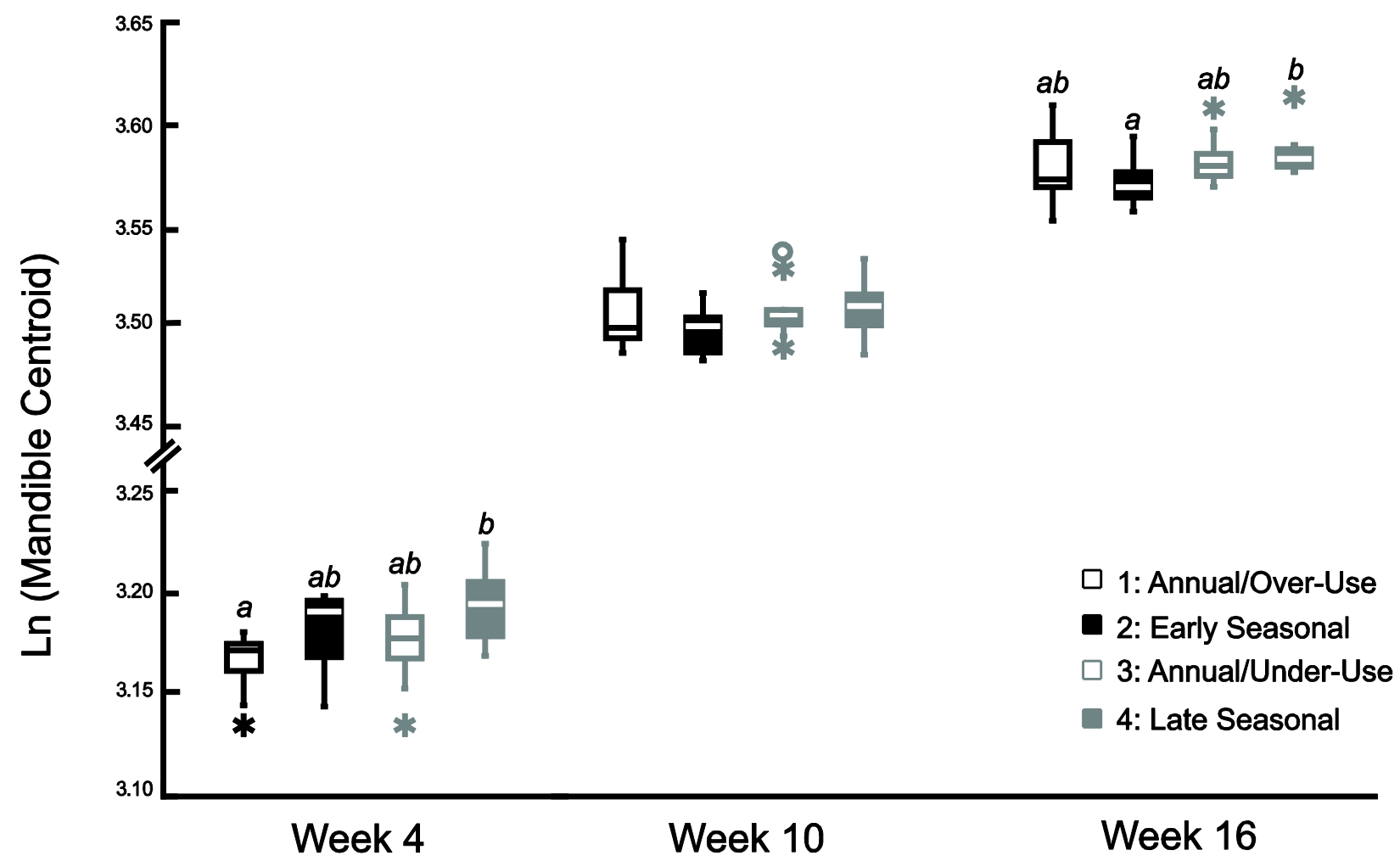

Fig. 2. Ln-transformed centroid sizes for the right hemimandible by cohort and ontogenetic stage. Cohorts with different superscript letters are significantly different $(p$ $<0.017)$. No significant difference was found among cohorts during week 10 . See Table S1 for cohort means and sample sizes, and Table S2 for pairwise $p$-values. 


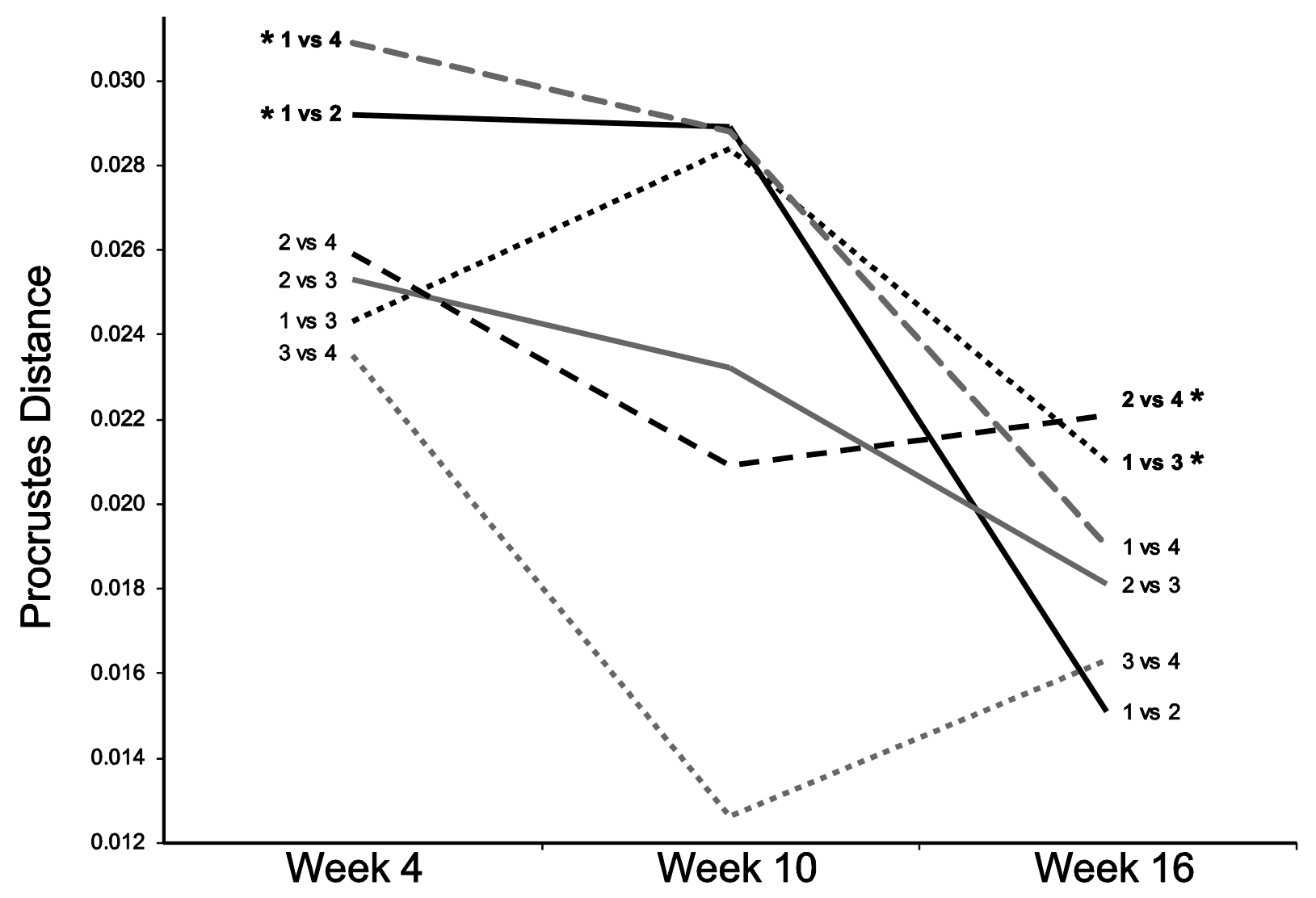

Fig. 3. Procrustes distances of mean shape $\left({ }^{*} p<0.017\right)$ between cohort pairs for the right hemimandible. See Table S3 for pair-wise $p$-values. 


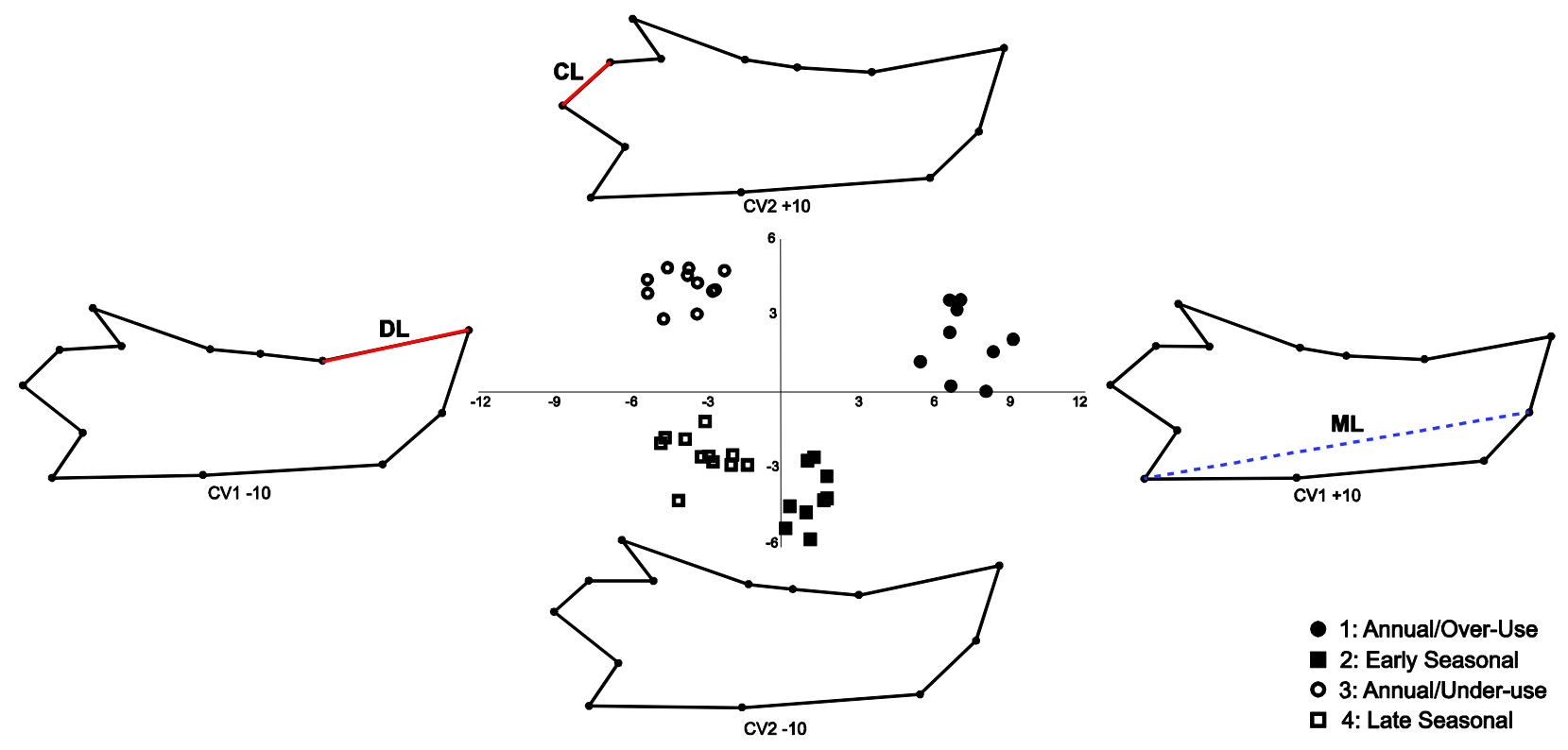

Fig. 4. A CVA of mandibular morphology in weanling rats (week 4). The X-axis is canonical variate 1 (CV1), the $\mathrm{Y}$-axis is CV2. CV1 maximizes the distance among cohorts using morphological variation pre-existing to the study, and describes differences in mandibular length, diastema length, and mandibular condyle length. Wireframe mandibles represent the shape changes at either $+10 /-10$ along their respective CVs. Solid red lines indicate an increasing linear or angular dimension; dashed blue lines represent a decreasing linear or angular dimension. See Table 3 for variable abbreviations. 

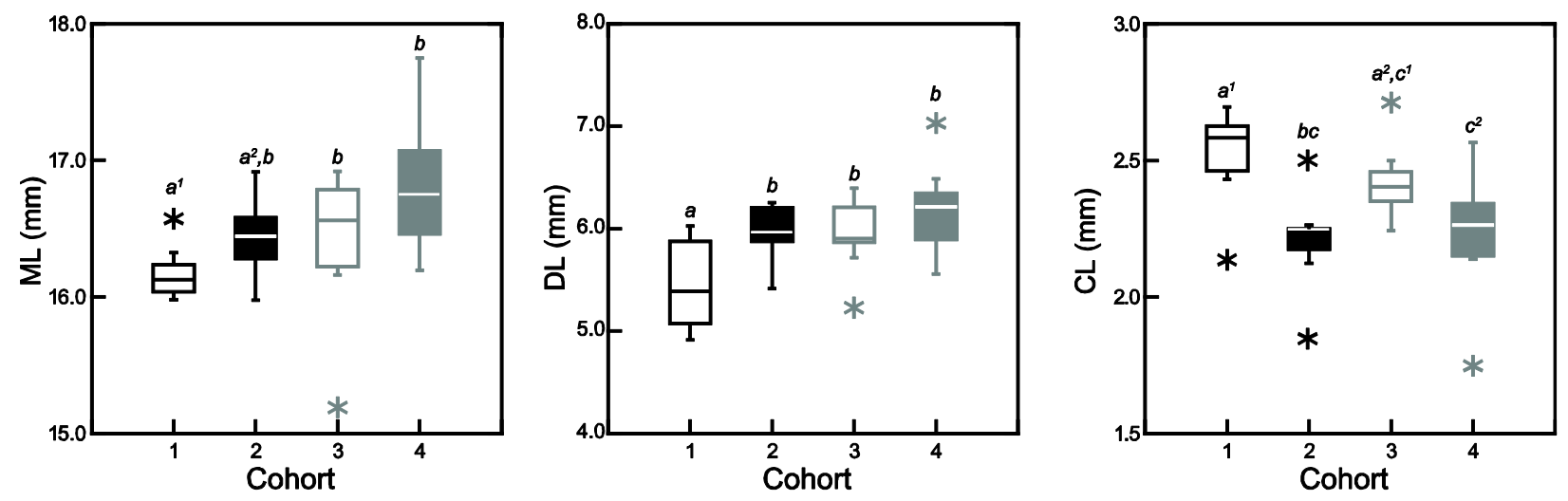

Fig. 5. Comparisons of linear interlandmark distances among cohorts during week 4 . Cohorts with different letters are significantly different $(p<0.017)$. Cohorts with different superscript numbers are separated by the morphometric variable in CV space but are not significantly different $(0.050<p<0.017)$. See Table 3 for variable abbreviations and Table S4 for pairwise $p$-values.
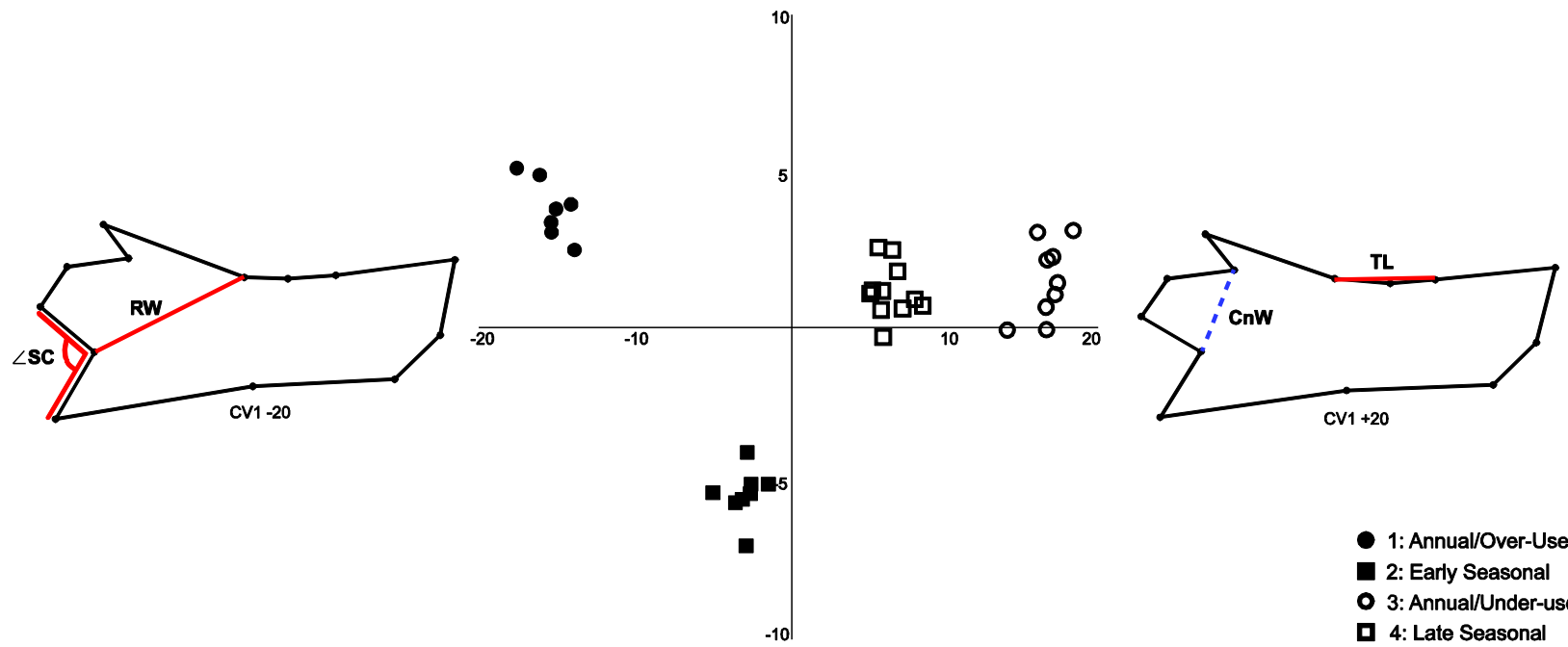

- 1: Annual/Over-Use

2: Early Seasonal

- 3: Annual/Under-use

(1: Late Seasonal

Fig. 6. A CVA of mandibular morphology in adolescent rats (week 10). The X-axis is canonical variate 1 (CV1), the Y-axis is CV2. CV1 groups individuals by their early (post-weaning) diet and describes differences in mandibular ramus size and tooth row length. Wireframe mandibles represent the shape changes at either $+20 /-20$ along their respective CVs. Solid red lines indicate an increasing linear or angular dimension; dashed blue lines represent a decreasing linear or angular dimension. See Table 3 for variable abbreviations. 

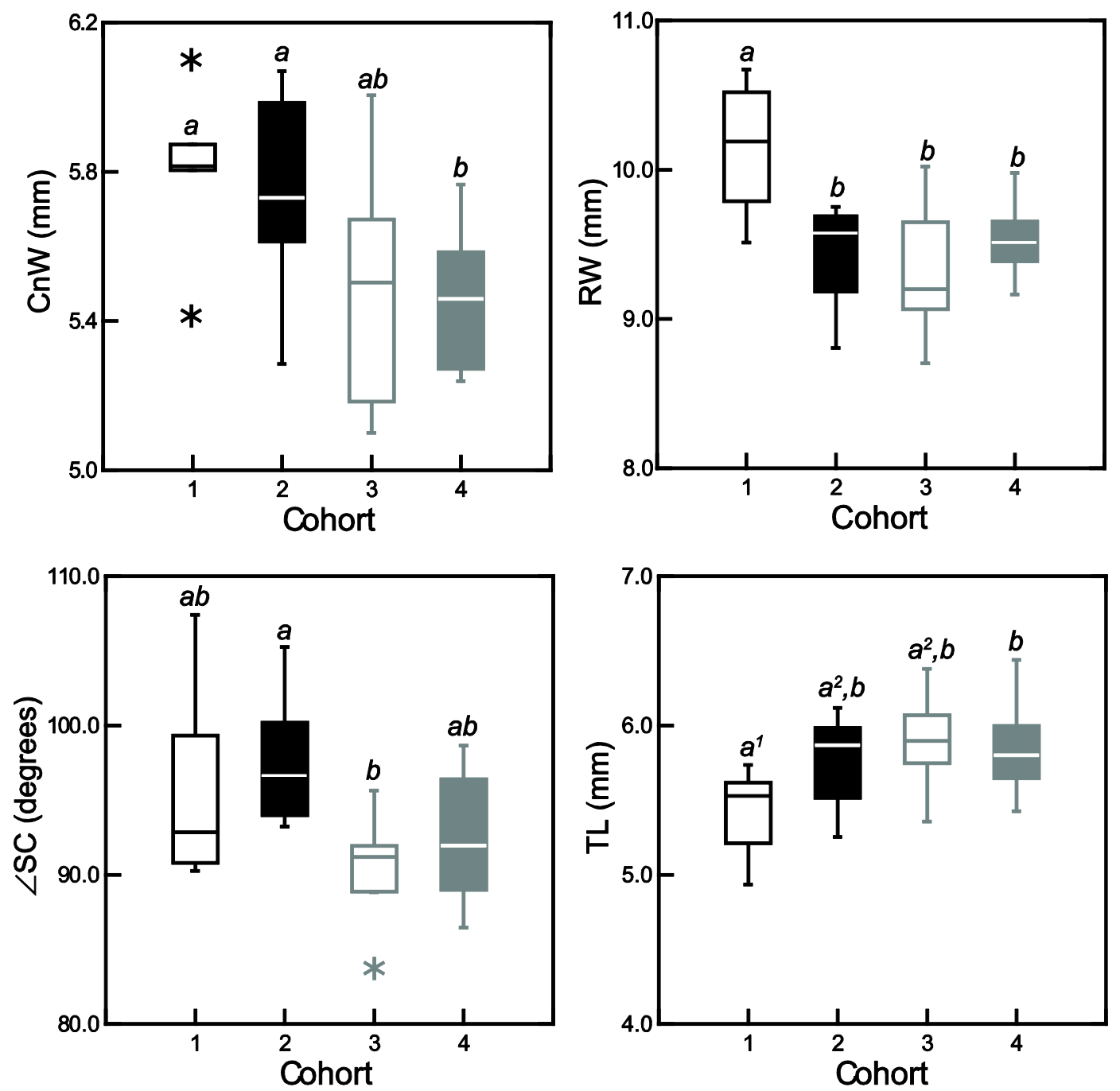

Fig. 7. Comparisons of linear interlandmark distances among cohorts during week 10. Cohorts with different letters are significantly different $(p<0.017)$. Cohorts with different superscript numbers are separated by the morphometric variable in CV space but are not significantly different $(0.050<p<0.017)$. See Table 3 for variable abbreviations and Table S5 for pairwise $p$-values. 


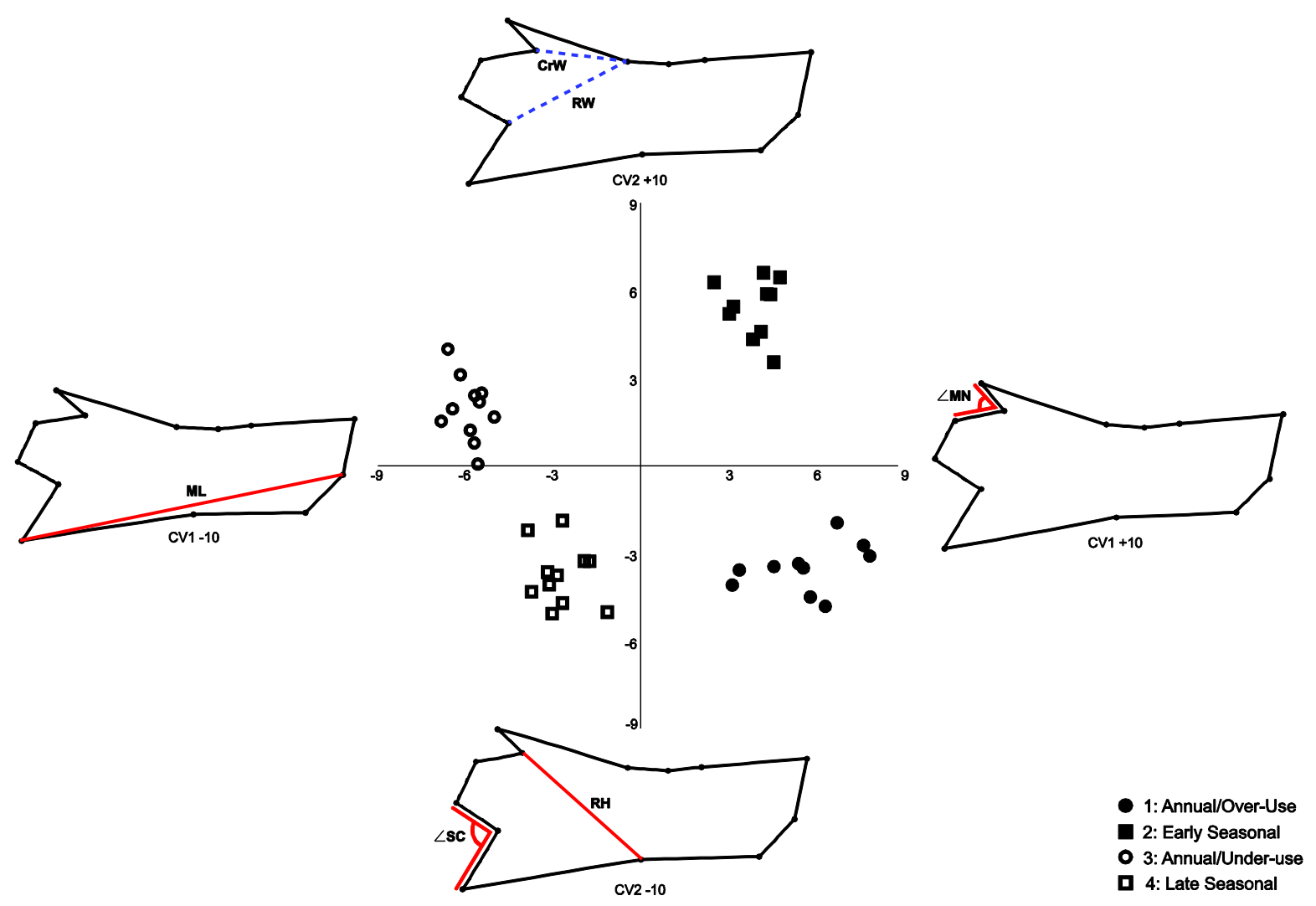

Fig. 8. A CVA of mandibular morphology in adult rats (week 16). The X-axis is canonical variate 1 (CV1), the $\mathrm{Y}$-axis is $\mathrm{CV} 2$. CV1 groups individuals by their early diet and describes differences in mandibular length and coronoid process morphology. CV2 groups individuals by their late diet and describes changes in angular process and masseteric fossa morphology. Wireframe mandibles represent the shape changes at either $+10 /-10$ along their respective CVs. Solid red lines indicate an increasing linear or angular dimension; dashed blue lines represent a decreasing linear or angular dimension. See Table 3 for variable abbreviations. 

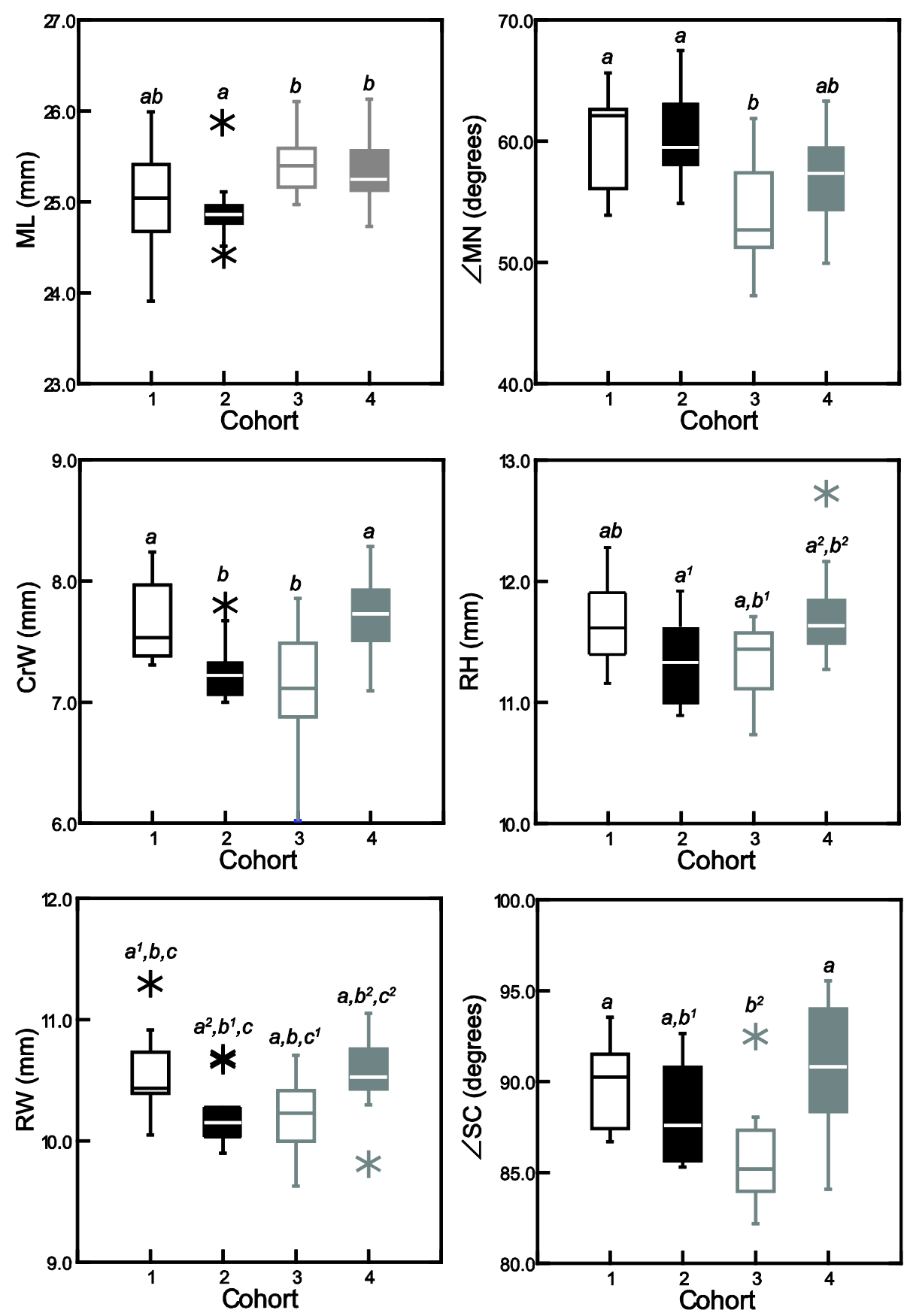

Fig. 9. Comparisons of linear interlandmark distances among cohorts during week 16 . Cohorts with different letters are significantly different $(p<0.017)$. Cohorts with different superscript numbers are separated by the morphometric variable in CV space but are not significantly different $(0.050<p<0.017)$. See Table 3 for variable abbreviations and Table 66 for pairwise $p$-values. 


\section{TABLES}

Table 1. Experimental design, including dietary treatment groups and the timing of dietary shifts.

\begin{tabular}{|l|l|l|}
\hline Cohort & $\begin{array}{l}\text { Weaning to adolescence } \\
\text { (day 22-64/week 4-10) }\end{array}$ & $\begin{array}{l}\text { Adolescence to adulthood } \\
\text { (day 65-110/week 10-16) }\end{array}$ \\
\hline $\begin{array}{l}\text { 1: Annual over- } \\
\text { ( } n=10)\end{array}$ & Solid pellets & Meal/powdered pellets \\
\hline $\begin{array}{l}\text { 2: Early seasonal } \\
(n=10)\end{array}$ & Solid pellets & \\
\hline $\begin{array}{l}\text { 3: Annual under- } \\
\text { use }\end{array}$ & Meal/powdered pellets \\
$(n=11)$ & \multicolumn{2}{|l}{} \\
\hline $\begin{array}{l}\text { 4: Late seasonal } \\
(n=11)\end{array}$ & Meal/powdered pellets & Solid pellets \\
\hline
\end{tabular}


Table 2. Landmarks of the right hemimandible used in the morphometric analyses. Landmarks are illustrated in Fig. 1.

\begin{tabular}{|l|l|}
\hline Landmark & Description \\
\hline 1 & Caudal TMJ \\
\hline 2 & Rostral TMJ \\
\hline 3 & Coronoid process \\
\hline 4 & Angular process \\
\hline 5 & Mandibular notch \\
\hline 6 & Subcondylar notch \\
\hline 7 & Preangular notch \\
\hline 8 & Superior incisal alveolus \\
\hline 9 & Inferior incisal alveolus \\
\hline 10 & Junction of the mandibular ramus and corpus \\
\hline 11 & Mesial M1 \\
\hline 12 & Mesial M2 \\
\hline 13 & Incisal ramus \\
\hline
\end{tabular}


Table 3. Morphometric variables discussed in Section 3, calculated as the angle or Euclidian distance between landmarks.

\begin{tabular}{|l|l|l|l|l|}
\hline \multicolumn{2}{|l|}{ Variable } & \multicolumn{3}{|l|}{ Landmarks } \\
\hline$\angle \mathrm{MN}$ & Mandibular notch angle & 1 & 5 & 3 \\
\hline$\angle \mathrm{SC}$ & Mabcondylar angle & 2 & 6 & 4 \\
\hline $\mathrm{CL}$ & Condylar process width & 5 & 6 & \\
\hline $\mathrm{CnW}$ & Coronoid process width & 4 & 10 & \\
\hline $\mathrm{CrW}$ & Diastema length & 11 & 8 & \\
\hline $\mathrm{DL}$ & Mandibular length & 4 & 61 & \\
\hline $\mathrm{ML}$ & Ramus height & 5 & 7 & \\
\hline $\mathrm{RH}$ & Ramus width & 6 & 10 & \\
\hline $\mathrm{RW}$ & Toothrow length & 10 & 11 & \\
\hline $\mathrm{TL}$ & & & 2 & \\
\hline
\end{tabular}

